\title{
IDONEIDAD DEL ESTABLECIMIENTO DEL ÁREA NATURAL PROTEGIDA DEL MAR PACÍFICO TROPICAL
}

\section{SUITABILITY OF THE ESTABLISHMENT OF THE PROTECTED NATURAL AREA OF THE TROPICAL PACIFIC SEA}

\author{
José Diego Hopkins Alfaro \\ Maestro of Science en Sostenibilidad Ambiental, Económica y Social \\ Estudiante de PhD in Environmental Research - Imperial College, London (UK) \\ d.hopkins17@imperial.ac.uk \\ Reino Unido, Londres \\ Karen E. Makuch \\ Imperial College London \\ k.e.makuch@imperial.ac.uk \\ Reino Unido, Londres \\ Zen Makuch \\ Imperial College London \\ z.makuch@imperial.ac.uk \\ Reino Unido, Londres
}

\section{SUMARIO}

- Introducción

- Idoneidad de Área Marina Protegidas

- Área Marina Protegida del Mar Pacífico Tropical

- Ley que declara interés nacional de AMP Mar Pacífico Tropical

- Establecimiento de AMP respecto del ordenamiento nacional e internacional

- Conclusiones

\section{RESUMEN}

En el presente ensayo analizaremos la idoneidad del establecimiento del Área Marina Protegida (AMP) del Mar Pacífico Tropical, que estaría ubicada frente a las costas de Piura y Tumbes. A partir de un análisis de la literatura respecto al diseño e implementación de AMPs, señalaremos algunos aspectos que deben ser tomados en cuenta por los administradores y tomadores de decisiones en todas las etapas referidas al proceso de asignación de la figura de AMP sobre determinada área geográfica. Asimismo, repasaremos la documentación oficial elaborada en el proceso de propuesta por diversos organismos estatales. El objetivo del presente ensayo es brindar luces respecto a la correcta elaboración de una propuesta que cambie el ordenamiento legal sobre el cual
Descansa el área geográfica elegida para ser protegida.

\begin{abstract}
In this essay we will analyze the suitability of the establishment of the Marine Protected Area (MPA) of the Tropical Pacific Sea, which would be located off the coast of Piura and Tumbes. Based on an analysis of the literature regarding the design and implementation of MPAs, we will point out some aspects that should be taken into account by administrators and decision makers in all the stages related to the process of assigning the MPA figure to a specific geographical area. Also, we will review the official documentation developed in the proposal process by various state agencies. The objective of this essay is to provide insights into the correct preparation of a proposal that changes the legal system on which the geographical area chosen to be protected rests.
\end{abstract}

\section{PALABRAS CLAVE}

Área Marina Protegida; Mar Pacífico Tropical; Recursos naturales; Recursos marinos.

\section{KEYWORDS}

Marine Protected Area; Tropical Pacific Sea; Natural Resources; Marine Resources. 


\section{INTRODUCCIÓN}

A inicios del año 2016, el Servicio Nacional de Áreas Naturales Protegidas por el Estado (SERNANP) propuso la creación de la Zona Reservada Mar Pacífico Tropical, al norte del Perú, la cual abarcaba un área de 116,139.95 hectáreas. Esta propuesta comprendería cuatro zonas prioritarias: La isla Foca, El Ñuro, los arrecifes de Punta Sal, y el Banco de Máncora.

Esta Zona Reservada es un estatus temporal que se le otorga a un área sobre la cual se pretenden desplegar mecanismos de protección ambiental. Sin perjuicio de su establecimiento, queda abierta la posibilidad de que esta misma área sea designada como un área natural protegida, variando el carácter temporal de protección a uno permanente.

La propuesta de establecer la Zona Reservada Mar Pacífico Tropical finalmente no prosperó, por lo cual, el SERNANP planteó la declaración directamente de área natural protegida, que en este caso trataremos como AMP dada su ubicación. Para ello, mediante Resolución Ministerial $\mathrm{N}^{\circ}$ 160-2017-MINAM, se conformó un grupo de trabajo sectorial encargado de recabar y analizar estudios complementarios para sustentar el establecimiento de la AMP.

Las áreas marinas protegidas (AMP) han sido definidas por la Unión Internacional para la Conservación de la Naturaleza (UICN) como: "Espacios geográficamente definidos, reconocidos, dedicados y manejados a través de medios legales entre otros, para alcanzar la conservación de la naturaleza a largo plazo junto con servicios ambientales y valores culturales asociados" (2012, página 2).

Los esfuerzos del SERNANP por promover la conservación de los recursos biológicos, hábitats y servicios ecosistémicos marinos son de resaltar. Sin embargo, en esta oportunidad dichas medidas se superponen a concesiones de exploración y explotación de petróleo otorgadas por el estado peruano con anterioridad, afectando de esta manera a derechos preexistentes que ostentan empresas petroleras.

Dentro de estas empresas petroleras encontramos a Karoon, a la cual se le otorgaron derechos de exploración y explotación en el lote petrolero Z38, el cual se encuentra superpuesto al Banco de Máncora, área ubicada a más de 40 millas de la costa peruana, y que forma parte de la propuesta de AMP planteada por SERNANP.

En el presente trabajo, haremos incidencia sobre mencionado lote petrolero, puesto que se constituye como un interesante objeto de estudio sobre el cual podemos desarrollar un análisis más profundo que nos lleve a señalar y resaltar los elementos que deben ser evaluados antes y durante el diseño e implementación de una AMP.

Debido a lo mencionado se nos vuelve a situar en el escenario de una aparente bifurcación entre el camino del desarrollo económico y el de conservación del medio ambiente. Planteamos que no siempre el camino lleva a una bifurcación, sino a una convergencia.

\section{IDONEIDAD DE ÁREAS MARINA PROTEGIDAS}

Actualmente, dada la atención puesta por la conservación de recursos naturales, observamos que algunos países se enfocan en la meta final de conservación soslayando otros aspectos importantes para su mantenimiento en el mediano y largo plazo. Podemos mencionar que la premura de Canadá por alcanzar las metas Aichi del Plan Estratégico para la Diversidad Biológica 20112020, dentro del marco de obligaciones del Convenio de Diversidad Biológica, hace crítico el hecho de asegurarse que los administradores de las AMP utilicen todo el conocimiento disponible para establecer AMPs que son legítimas, efectivas, y que puedan producir genuinos resultados de conservación (Dehens \& Fanning, 2018).

Esta premura hace hecho que muchas AMP alrededor el mundo se hayan caracterizado por ser papermarks, es decir, áreas legalmente designadas pero que hacen muy poco por la conservación. De hecho, se ha estimado que sólo $31 \%$ de las AMP son efectivas globalmente, con la mayoría fracasando en el intento por alcanzar las metas y objetivos establecidos (Pomeroy, Watson, Parks, $\&$ Cid, 2005). Esto ha sido en parte atribuido a una inadecuada consideración de la dimensión social asociada a la implementación de la AMP.

De cualquier modo, es importante anotar que algunas AMPs son utilizadas como vehículo para alcanzar el cumplimiento de metas 
globales de conservación marina, y como tales contribuyen más a objetivos políticos que a fortalecer la conservación de la biodiversidad (Ban et al., 2017). Por ello, el establecimiento de estas áreas debe obedecer a criterios técnicos y científicos rigurosos para que no simplemente se busquen crear políticas ambientales no dirigidas a lograr un beneficio fáctico en cuestiones biológicas, además de considerar los espectros sociales y económicos de la comunidad.

La designación de espacios geográficos bajo la figura de AMP busca una multiplicidad de resultados que van más allá del objetivo ambiental. Se espera que las áreas naturales protegidas satisfagan tanto necesidades biológicas como socioeconómicas, lo cual significa que el éxito de una AMP no se ampara sólo en la seguridad institucional prolongada ni el estatus legal establecido, sino que debe tener en cuenta resultados ecológicos, sociales, políticos y económicos (Speed \& Levine, 2014). Esta atención, que debe ser puesta sobre diversos aspectos de la comunidad en la cual se establecen estos espacios protegidos, podría determinar el éxito o fracaso de la AMP.

Resulta importante comprender el contexto para asegurarse que las iniciativas enlazan con las necesidades y cultura de la comunidad, que la intervención está siendo implementada en la locación correcta y apunta a los stakeholders adecuados (Katikiro, 2016). Es preciso señalar que al referirnos a stakeholders no nos limitamos a los pobladores de comunidades directamente relacionadas con la AMP, sino también a organizaciones y empresas que puedan verse afectadas por el establecimiento de esta área de protección, todo ello en pos de mantener el estatus de conservación en el largo plazo.

La consideración de los pobladores en las zonas aledañas al área designada bajo protección es sumamente importante al momento de diseñar e implementar la AMPs. Citando algunos ejemplos, podemos señalar que se ha demostrado que las AMPs en Filipinas e Indonesia fueron éxitos biológicos, pero fracasos sociales debido a la limitada participación, un inequitativo repartimiento de beneficios económicos, y una ausencia de mecanismos de resolución de conflictos (James

\& Dearden, 2014). Todas estas características determinan el éxito o fracaso del área protegida, pero todas se relacionan con los stakeholders involucrados, lo cual hace determinante su atención.

Existen herramientas que deben ser utilizadas por administradores de la AMP al momento de diseñar e implementar la zona bajo protección. Entender preferencias sociales, desarrollar indicadores referentes a ecosistemas, conducir análisis de riesgo, y evaluar distintos escenarios de manejo, es fundamental para una exitosa conducción de la AMP (Levin \& Mo, 2014). Debe entenderse que el adecuado manejo de una AMP no se limita a la implementación, sino que debe partir desde el diseño.

En concordancia con las metas globales acordadas, el número de AMPs está creciendo rápidamente, aunque los beneficios socioeconómicos aún son difíciles de predecir (Edgar et al., 2014). Estos beneficios socioeconómicos, de no ser generados por la asignación de la categoría de AMP a determinada área geográfica, por lo menos no deberían ser anulados por el establecimiento de esta. Es decir, si la AMP no genera beneficios para la comunidad, como mínimo se debería evaluar si esta no anulará los beneficios que la comunidad ya está recibiendo.

\section{Área Marina Protegida del Mar Pacífico Tropical}

En la propuesta para la constitución de la AMP del Mar Pacífico Territorial (Fig. 1) se contemplan las distintas áreas ya mencionadas, dentro de las cuales encontramos al Banco de Máncora, ubicada a más de cuarenta millas de la costa, constituyéndose como el área más alejada de la propuesta. Esta área se superpone con el lote petrolero Z-38 (Fig.2), adjudicado a la empresa Karoon.

En el caso del establecimiento de la zona del Banco de Máncora, no queda debidamente establecida la idoneidad de su inclusión dentro de las áreas que conformarían la AMP del Mar Pacífico Tropical. La efectividad de grandes y remotas AMPs para conservar la biodiversidad no queda del todo clara, sobre todo cuando las aguas costeras cercanas a centros urbanos están más expuestas a las presiones antropogénicas, y por lo tanto necesitan mayor protección (Moore et al., 2016). Por ello, la lejanía y aislamiento del Banco de Máncora requiere señalar los criterios científicos utilizados para su inclusión. 
FIGURA 1.- Zona Reservada Mar Pacífico Tropical (SERNANP)

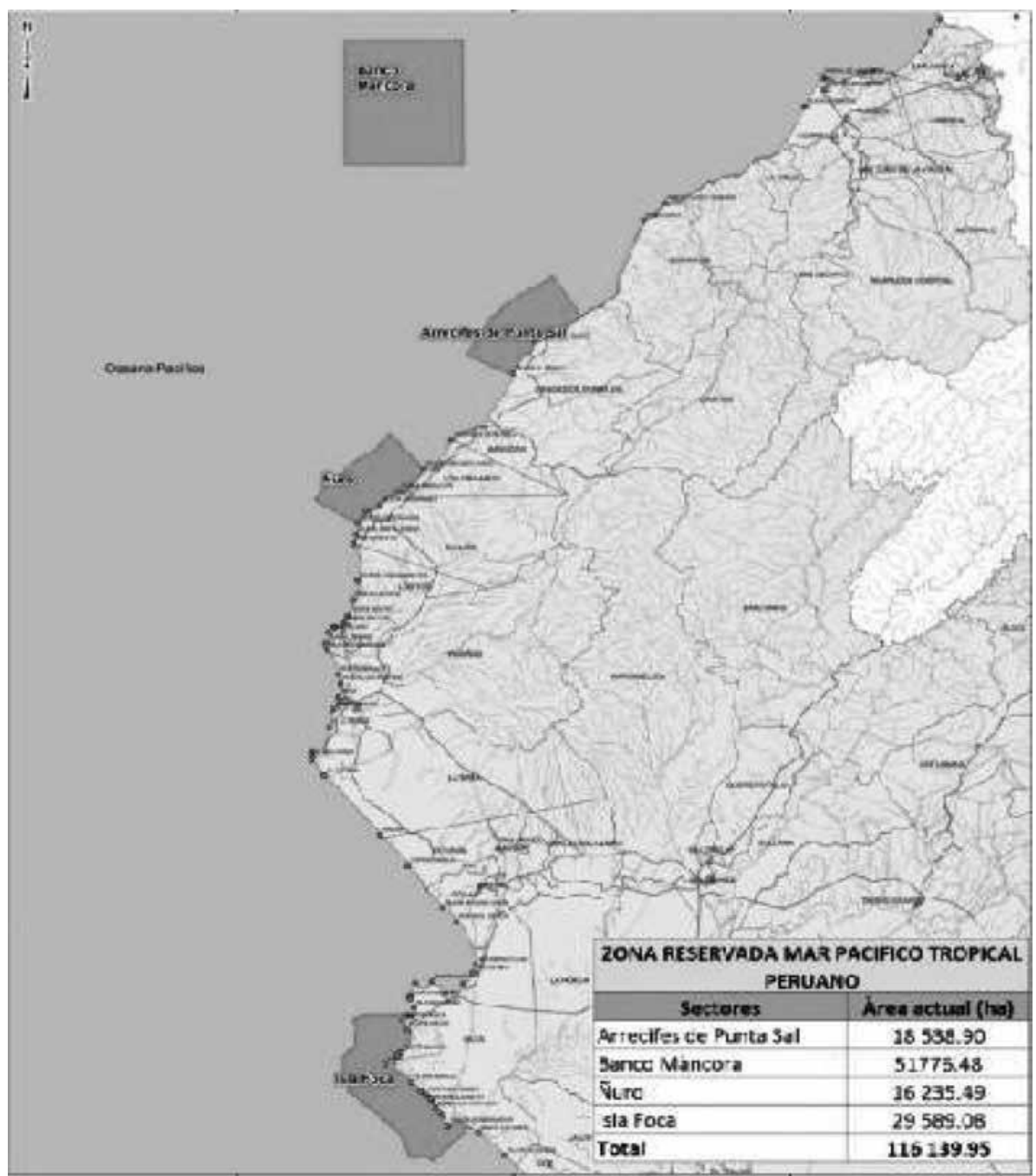

Fuente y elaboración:http://www.minam.gob.pe/comuma/wp-

content/uploads/sites/106/2016/06/10.SERNANP_M.Pastor_-Foro-OC\%C3\%89ANOS-SOSTENIBLES_ZR-Mar-PacificoTropical_08Jun2016.pdf 


\section{FIGURA. 2.- Lotes petroleros concesionados (modificado de PeruPetro)}

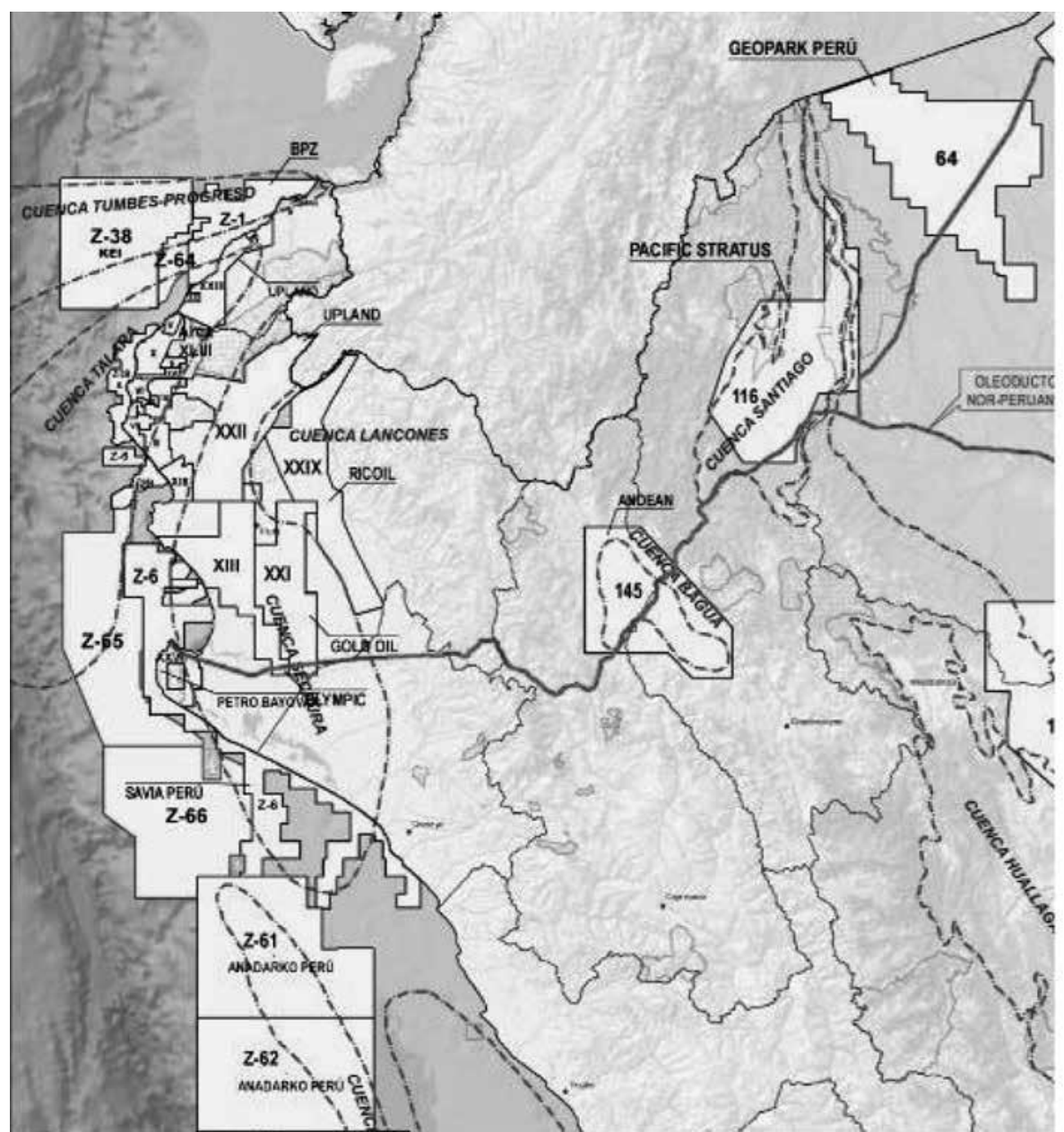

Fuente y elaboración: http://www.perupetro.com.pe/wps/wcm/connect/corporativo/1ac7d4da-d2cd-410d-b178f706a4977eda/1/CNT+Web_Octubre_2018.jpg?MOD=AJPERES\&CACHEID=ROOTWORKSPACE.Z18_N2E4HH41J8RPE0AF8M8T5Q3K Q31ac7d4da-d2cd-410d-b178-f706a4977eda/1

De hecho, el biólogo Yuri Hooker, del Laboratorio de Biología Marina de la Universidad Peruana Cayetano Heredia, señaló que cuando la propuesta original fue presentada en el año 2011, no se incluía al Banco de Máncora, pues había poca información sobre biodiversidad en comparación del resto de zonas.

Efectivamente, el Instituto del Mar del Perú (IMARPE), mediante oficio $\mathrm{N}^{\circ}$ 2016-2016IMARPE/DEC, realiza una evaluación del Informe Técnico presentado por parte del SERNANP con oficio $\mathrm{N}^{\circ}$ 143-2016-SERNANP-J, por el cual se remitía al Ministerio de la Producción la propuesta de la Zona Reservada Mar Pacífico Tropical Peruano.

La evaluación realizada por IMARPE señala que la propuesta no describe los criterios utilizados por SERNANP para identificar las áreas con potencial de conservación, así como la delimitación de las mismas. Se indica que los límites fueron realizados en base a imágenes satelitales de Google Earth, no indicando metodología ni criterios ecosistémicos. También se señala que no se especifica claramente la conectividad entre las zonas seleccionadas para establecerlas como un subsistema. Ello devela que las áreas 
que no han sido determinadas obedeciendo criterios científicos para evaluar de manera certera los requerimientos de protección, y las consecuencias económico-sociales del establecimiento de esta AMP.

Más allá de las falencias científicas para el establecimiento del área mencionada, se debe poner más atención a la incorporación de conocimiento de las comunidades. Esto requerirá que la administración mejore el vínculo con los stakeholders (Li \& Fluharty, 2017). Esto podrá hacer que la administración ambiental pueda establecer un mapa situacional más claro que permita evaluar de manera más precisa los requerimientos para el sostenimiento de la AMP.

Para ello se deben amalgamar criterios científicos y económico-sociales, sobre todo cuando en la propuesta se hace mención de que los recursos marinos están siendo explotados por la pesca ilegal, de manera que se pone en peligro la sostenibilidad ambiental de la zona, afectando espectros biológicos y sociales, siendo ello motivo para la constitución de la AMP.

Sin embargo, tal cual lo señala el informe de IMARPE, el establecimiento de AMP, como lo han sido la Reserva Nacional de Paracas e Isla San Fernando, no han solucionado la problemática de la pesca ilegal en estas zonas, por lo cual, el establecimiento de la zona reservada no es una silver bullet que pueda solucionar los problemas que afectan a la conservación de los recursos marinos dentro del área propuesta. Cabe resaltar que, de la experiencia de la Dirección General de Supervisión y Fiscalización del Viceministerio de Pesca y Acuicultura, se da cuenta que la creación de AMPs no asegura que no se realicen actividades de pesca ilegal.

El establecimiento de la AMP no sólo afectaría los derechos preexistentes de la empresa petrolera Karoon, sino que también se afectarían a 5,566 armadores artesanales de la Región Piura, así como 1,138 de la Región Tumbes, generando un gran impacto en el bienestar económico-social de la comunidad.

No todos los involucrados se ven afectados de la misma manera a causa de intervenciones como las AMPs. Pueden producirse diversos impactos y compensaciones entre grupos con el transcurrir del tiempo. No solo soluciones efectivas requieren la observancia del contexto particular, historia local, y valores culturales y costumbres, sino también requieren una apreciación de las diversas necesidades, intereses y características de los stakeholders involucrados (Chaigneau \& Brown, 2016). Son ellos finalmente, pobladores, organizaciones, empresas, y autoridades, quienes dotarán de legitimidad y estabilidad a la AMP en el mediano y largo plazo.

Asimismo, el establecimiento de la AMP, acarrea consecuencias científicas y sociales en cuanto a temas de conservación. Debemos considerar la figura del spillover, definido como el movimiento de peces a través de los límites de la reserva hacia un área de pesca, la cual se espera que ocurra, sobre la base de los principios fundamentales del movimiento aleatorio (Buxton, Hartmann, Kearney, \& Gardner, 2014). Cabe señalar que diversos estudios han demostrado el spillover de abundante biomasa de adultos de especies marinas desde la reserva, y el mantenimiento de capturas cerca de los bordes de la reserva (Uss, 2011).

Ello es importante puesto que el spillover de especies marinas adultas a los bordes de la reserva podría generar que pescadores que no son de la zona en donde se ha implementado la AMP acudan a realizar sus capturas a los bordes de esta, pudiendo producir conflictos sociales con los pobladores de las comunidades aledañas. Este fenómeno conocido como "fishing-the-line" alrededor de las AMPs ha sido ampliamente documentado, y permite a los pescadores beneficiarse del spillover de valiosas especies comerciales (Leary et al., 2018).

Si bien es cierto, el establecimiento de zonas de protección marinas obedece a diversos objetivos planteados por la autoridad competente, estos esfuerzos no deben limitarse a la creación de las AMPs, sino que tienen que crearse las condiciones necesarias para hacerlas sostenibles. Con el fin de estimular la conservación de biodiversidad marina y el desarrollo socioeconómico, los esfuerzos de intervención deben pasar de ser simples ayudas a ser asociaciones de desarrollo sostenible con responsabilidad compartida entre donantes y beneficiarios (Katikiro, 2016). Dichas asociaciones pueden tener como eje principal a los pobladores y a las empresas. 


\section{LEY QUE DECLARA INTERÉS NACIONAL DE AMP MAR PACÍFICO TROPICAL}

El Congreso de la República, a través de la Comisión de Pueblos Andinos, Amazónicos y Afroperuanos, Ambiente y Ecología, aprobó el dictamen recaído en los proyectos de ley 1087/2016-CR y 1143/2016-CR, declarando de interés nacional la conservación y protección del ecosistema marino de la Isla Foca, El Ñuro, Los Arrecifes de Punta Sal, y el Banco de Máncora, en los departamentos de Piura y Tumbes, y su establecimiento como Área Natural Protegida Mar Pacífico Tropical Peruano.

Pese a ser una ley meramente declarativa, ya que el establecimiento de áreas naturales de protección cuenta con un proceso diferente, es oportuno señalar que en el dictamen mencionado tampoco se siguieron procedimientos técnicos, ni estudios socioeconómicos que permitan fundamentar lo declarado en sus conclusiones.

Pese a que el Manual de Técnica Legislativa establece el análisis costo-beneficio de la ley propuesta, lo cual implica identificar a los actores afectados de manera positiva o negativa con su aprobación, la Comisión sencillamente indica:

\begin{abstract}
los actores que resultan afectados positivamente con la norma son los pobladores que habitan cerca al grupo de islas que conforman el Mar Pacífico Tropical Peruano, por la protección que recibirán los recursos naturales y diversidad biológica del mismo, y por la promoción de actividades turísticas que mejorarán la actividad económica de dicha zona. (2017, Página 24)
\end{abstract}

Sin embargo, no se realiza ningún tipo de evaluación económica con ingresos proyectados.

Asimismo, continúa:

podrían nombrarse como actores afectados negativamente con la norma a las empresas de hidrocarburos o de gas cuyas inversiones estén en la zona. Sin embargo, ello no resulta admisible dado que la Ley 26834 garantiza los derechos otorgados por el Estado a particulares. La conservación y protección de recursos naturales y diversidad biológica no pueden, ni deben ser incompatibles con las actividades que hemos referido. (2017, Página 24)

En este punto es necesario señalar que se deben tener muy en cuenta que las actividades sí podrían devenir en incompatibles en el caso de que se apruebe el establecimiento de la AMP Mar Pacífico Tropical Peruano, pues una vez asignado este estatus, todas las actividades que se desarrollen dentro de la AMP deberán obedecer a su Plan Maestro, el cual podría considerar incompatible con el desarrollo de la AMP a las actividades de explotación de petróleo, por lo que éstas quedarían prohibidas de realizarse.

Las acciones de conservación deben anticipar la adjudicación de concesiones de exploración y explotación del mismo modo que se debe hacer para la industria del petróleo y del gas, pues estas áreas probablemente no cuentan con una adecuada evaluación biológica (Vaz, Baez, \& Fraga, 2017). El ampararse en una evaluación no realizada en el momento adecuado, previo a la adjudicación de la concesión, atentaría contra la estabilidad jurídica de las inversiones realizadas para la implementación del proyecto de concesión. Ello no impide que se realicen acciones expost con el objetivo de mejorar las condiciones biológicas de la zona por parte de las autoridades ambientales, todo ello sin afectar derechos preexistentes.

Es fundamental considerar la información disponible, no sólo para diseñar e implementar el proyecto de área marina protegida, sino que también tiene que considerarse como herramienta para anticipar potenciales situaciones. En este caso, cabe indicar que para el año 2035, la demanda de petróleo está proyectada en crecer un $30 \%$, gas natural 53\%, y carbón un 50\% (Seabrook \& Possingham, 2015), por lo cual este incremento en la demanda de mencionadas fuentes de energía tiene que ser evaluado con el objetivo de generar una mejor propuesta para el área a considerar bajo protección.

Del mismo modo, la evaluación de distintos aspectos de las AMPs es relevante al momento de medir el impacto positivo generado por estas en las comunidades aledañas. Esta evaluación también nos puede arrojar información sobre aspectos críticos que necesitan ser tratados. 
En diversos estudios, la seguridad legal, relacionada al estatus legal del área protegida se vio afectada por la ausencia de delimitación claramente establecida, y conflictos no resueltos respecto a la propiedad del área (Araújo \& Bernard, 2016). Es aquí donde reside la importancia de base científica para la delimitación, y el involucramiento de todos los stakeholders para evitar potenciales conflictos que atenten contra la estabilidad de la AMP.

\section{ESTABLECIMIENTO DE AMP RESPECTO DEL ORDENAMIENTO NACIONAL E INTERNACIONAL}

Es preciso mencionar que, si bien es cierto lo tratados internacionales constituyen una fuente de derecho internacional, éstos establecen parámetros que deberán ser cumplidos por la comunidad en general. Sin embargo, la gran mayoría de éstos son pasibles de libre renunciabilidad, siendo únicamente exigibles de cumplimiento para los países que hayan ratificado mencionados instrumentos.

Los acuerdos internacionales como los citados en el presente trabajo de investigación plantean metas a alcanzar con el objetivo de preservar el medio ambiente, instando a las partes al debido cumplimiento del mismo. Cabe señalar que dichas metas deberán ser impulsadas a través de medidas bottom-up, lo cual constituye como base de la estrategia para el cumplimiento de los fines perseguidos por los tratados internacionales, a las medidas que cada Estado pueda implementar dentro de su propia jurisdicción.

En este orden de ideas, las medidas desplegadas por el Estado peruano están fuertemente vinculadas con lo establecido dentro de su ordenamiento nacional. Al respecto es preciso señalar, por ejemplo, que la Ley $\mathrm{N}^{\circ} 26834$, Ley de Áreas Naturales Protegidas, reconoce la condición de Patrimonio de la Nación y de Dominio Público de las Áreas Naturales, debiéndose mantener su estado natural a perpetuidad, lo cual no sólo fija un punto de partida respecto de aportes del estado peruano hacia la consecución de las metas mundiales, sino que también procura que dicho establecimiento inicial no pueda verse afectado en manera alguna en el futuro, perpetuando el aporte generado mediante el establecimiento del área natural.
Asimismo, la protección del medio ambiente se encuentra consagrada en la Constitución Política del Perú, la cual recoge en su artículo 68 la disposición que señala que el Estado está obligado a promover la conservación de la diversidad biológica y de las áreas naturales protegidas.

Es aquí que podemos hacer una clara distinción entre el establecimiento de las áreas naturales protegidas, y el establecimiento de concesiones mineras o de hidrocarburos dentro del territorio nacional. La diferencia radica en la exigibilidad del cumplimiento de las obligaciones que emanan de dicha condición.

En los casos en los que el Estado, en este caso el peruano, decida por cuestiones relacionadas al cambio de las políticas estatales el decantarse por la priorización de actividades distintas a las exclusivamente relacionadas con la conservación podrá realizarlo mediante cambios relacionados al plan maestro del área en cuestión, no implicando ello la eliminación del estatus de área natural protegida, como sí se da en diversos estados, siendo el caso más reciente el de Brasil, que ha procedido a la eliminación del estatus de once áreas naturales protegidas.

Sin embargo, la eliminación de las concesiones mineras o de hidrocarburos, al generar impactos económicos negativos a las empresas que hubieren recibido la concesión, por resultar afectadas, podrían hacer exigible el cumplimiento de las obligaciones asumidas por el Estado, ello ante la jurisdicción internacional, siendo pasible de la imposición de indemnizaciones o restablecimiento del estado de las cosas al previo al levantamiento de la concesión.

\section{CONCLUSIONES}

En el camino del desarrollo económico del país parece haber una bifurcación entre conservacionistas y empresas explotadoras de recursos naturales, e incluso parece no haber una concordancia gubernamental en cuanto a la protección del medio ambiente y el desarrollo de proyectos de inversión que exploten recursos naturales, puesto que de existir una política nacional de desarrollo sostenible se podría avanzar en cuanto al establecimiento de una posición única de estado respecto a estos temas. 
Existe la creencia que no es posible la protección ambiental si es que nos orientamos al desarrollo económico, y viceversa. Este es un escollo que debe ser superado mediante la promoción de acuerdos que beneficien mutuamente a ambas partes, los cuales pueden ser alcanzados luego de tomar en cuenta y sopesar cada factor relevante. Por ejemplo, Energy and Biodiversity Initiative (EBI) fue formada por compañías de energía (BP, ChevronTexaco, Shell, Statoil) y organizaciones conservacionistas (Kark, Brokovich, Mazor, \& Levin, 2015). La colaboración entre ambas posiciones se constituye como un elemento importante que puede coadyuvar a la estabilidad económica y social del establecimiento de la AMP respecto de la comunidad alrededor de la zona designada.

Asimismo, antes de plantear alguna propuesta de alguna área natural protegida en general o una AMP en particular, se deben exigir el seguimiento estricto de criterios técnicos que sean la base de su selección. Del mismo modo, la evaluación del contexto social y económico, incluyendo el tratamiento de todos los stakeholders involucrados, constituye un punto fundamental cuando se busca darle sostenibilidad al área protegida.

Cabe indicar que lo siguiente mejor a tener una amplia base de datos sobre la efectividad de las dimensiones biológicas y socioeconómicas de las AMPs (actualmente ausentes), es evaluar los objetivos de los administradores y tomadores de decisiones al momento de diseñar, clasificar e implementar una AMP (Horta et al., 2016). Por consiguiente, todo el proceso llevado a cabo para la designación de una AMP debe obedecer a criterios científicos, económicos, y legales para el diseño e implementación de una AMP, lo cual constituye la base de su sostenibilidad en el tiempo. Estos criterios deben asegurar la idoneidad geográfica, económica y social de la AMP, salvaguardando al mismo tiempo los derechos de todos los actores involucrados.

Sin embargo, podemos concluir que la exigibilidad del cumplimiento del establecimiento de una concesión minera o hidrocarburífera, y la exigibilidad del cumplimiento del establecimiento de un área natural protegida distan de gran manera entre sí, por lo cual podemos inferir el privilegio de la protección de explotación de recursos minerales e hidrocarburos, por sobre la protección de la conservación del medio ambiente a través de áreas naturales protegidas.

Más allá de lo anteriormente señalado, es de puntualizar que las prioridades del Estado pueden variar en el tiempo, por lo cual se requiere un análisis profundo de las razones del establecimiento de áreas naturales protegidas o el otorgamiento de concesiones para la explotación de recursos, observando las posibles consecuencias económicas y sociales que la variación a posteriori de cualquiera de los dos estados pudiera acarrear.

\section{FUENTES DE INFORMACIÓN}

\section{Fuentes bibliográficas}

Levin, P. S., \& Mo, C. (2014). Marine ecosystem regime shifts: challenges and opportunities for ecosystem-based management, 1-8.

Seabrook, L. M., \& Possingham, H. P. (2015). Biodiversity risks from fossil fuel extraction, 910.

Uss, G. A. R. R. (2011). Enhanced biodiversity beyond marine reserve boundaries: The cup spillith over, 21(1), 241-250.

\section{Fuentes electrónicas}

Araújo, J. L., \& Bernard, E. (2016). Management effectiveness of a large marine protected area in Northeastern Brazil. Ocean \& Coastal Management, 130, Páginas 43-49. https://doi. org/10.1016/j.ocecoaman.2016.05.009

Ban, N. C., Davies, T. E., Aguilera, Aguilar Stacy E., Brooks, C., Cox, M., Epstein, G., Evans, L. S., Maxwell, Sara M., Nenadovic, M. (2017). Social and ecological effectiveness of large marine protected areas. Global Environmental Change, 43, 82-91. https://doi. org/10.1016/j.gloenvcha.2017.01.003

Buxton, C. D., Hartmann, K., Kearney, R., \& Gardner, C. (2014). When Is Spillover from Marine Reserves Likely to Benefit Fisheries?. PLOS ONE, 9(9), 1-7. https://doi.org/10.1371/ journal.pone. 0107032

Chaigneau, T., \& Brown, K. (2016). Challenging the win-win discourse on conservation and development: analyzing support for marine protected áreas. Ecology and Society, 21(1). http://dx.doi.org/10.5751/ ES-08204-210136 
Dehens, L. A., \& Fanning, L. M. (2018). What counts in making marine protected areas ( MPAs ) count? The role of legitimacy in MPA success in Canada. Ecological Indicators, 86(March 2018), 45-57. doi.org/10.1016/j.ecolind.2017.12.026

https://

Day J., Dudley N., Hockings M., Holmes G., Laffoley D., Stolton S. \& S. Wells, (2012). Guidelines for Applying the IUCN Protected Area Management Categories to Marine Protected Areas. Gland, Switzerland. Recuperado de: https://cmsdata.iucn.org/

downloads/uicn_categoriesamp_eng.pdf

Edgar, G. J., Stuart-smith, R. D., Willis, T. J., Kininmonth, S., Baker, S. C., Banks, S., Barrett, Neville S., Becerro, Mikel A., Bernard, Anthony T. F., Berkhout, J., Buxton, Colin D., Campbell, Stuart J., Cooper, Antonia T., Davey, Marlene., and others (2014). Global conservation outcomes dependo $\mathrm{n}$ marine protected areas with five key features. Nature, 506, 216-220. https://doi.org/10.1038/ nature13022

Horta, B., Claudet, J., Franco, G., Erzini, K., Caro, A., \& Gonçalves, E. J. (2016). A regulation- based classi fi cation system for Marine Protected Areas (MPAs). Marine Policy, 72, 192- 198. https://doi.org/10.1016/j. marpol.2016.06.021

James, N., \& Dearden, P. (2014). Why local people do not support conservation: Community perceptions of marine protected area livelihood impacts, governance and management in Thailand. Marine Policy,

44, 107-116. https://doi.org/10.1016/j. marpol.2013.08.017

Kark, S., Brokovich, E., Mazor, T., \& Levin, N. (2015). Emerging conservation challenges and prospects in an era of offshore hydrocarbon exploration and exploitation. Conservation Biology, 29(6), 1573- 1585. https://doi. org/10.1111/cobi.12562

Katikiro, R. E. (2016). Improving alternative livelihood interventions in marine protected areas : A case study in Tanzania. Marine Policy, 70, 2229. https://doi.org/10.1016/j. marpol.2016.04.025

Leary, B. C. O., Ban, N. C., Fernandez, M., Friedlander, A. M., García-borboroglu, P., Golbuu, Y., ... Roberts, C. M. (2018).
Addressing Criticisms of Large-Scale Marine Protected Areas, 68(5), 359-370. https://doi. org/10.1093/biosci/biy021

Li, Y., \& Fluharty, D. L. (2017). Marine protected area networks in China: Challenges and prospects. Marine Policy, 85(July), 8-16. https://doi.org/10.1016/j.marpol.2017.08.001

Moore, C. H., Radford, B. T., Possingham, H. P., Heyward, A. J., Stewart, R. R., Watts, M. E., ... Berry, O. (2016). Improving spatial prioritisation for remote marine regions: optimising biodiversity conservation and sustainable development trade-offs, (August), 1-12. https://doi.org/10.1038/srep32029

Pomeroy, R. S., Watson, L. M., Parks, J. E., \& Cid, G. A. (2005). How is your MPA doing? A methodology for evaluating the management effectiveness of marine protected areas, 48, 485502.

ocecoaman.2005.05.004 https://doi.org/10.1016/j.

Congreso de la República del Perú (2017). Predictamen recaído en los proyectos de Ley 1087/2016-CR y 1143/2016-CR que, con texto sustitutorio, propone aprobar la Ley que declara de interés nacional la conservación y protección del ecosistema marino de la Isla Foca, El ñuro, los arrecifes de punta sal y el banco de máncora, en los departamentos de Piura y tumbes, y su establecimiento como área natural protegida mar pacífico tropical peruano. Recuperado del sitio de internet: http://www. congreso.gob.pe/Docs/comisiones2017/ Comision _ de _ Pueblos _ Andinos/files/ pl_1143/predictamen_1087_-1143.pdf

Speed, J., \& Levine, A. (2014). What makes a “ successful " marine protected area? The unique context of Hawaii ' s fi sh replenishment areas. Marine Policy, 44, 196-203. https://doi. org/10.1016/j.marpol.2013.08.022

Vaz, G., Baez, D. M., \& Fraga, A. (2017). Conservation of deep-sea ecosystems within offshore oil fi elds on the Brazilian margin, SW Atlantic. Biological Conservation,

206, 92-101. https://doi.org/10.1016/j. biocon.2016.12.026 